\title{
Overnight postoperative monitoring: necessity or not?
}

Dr. Peter Alley, a thoracic surgeon, was seeking IACUC approval for a postoperative drug treatment that had the potential to be toxic to rats, the animal model that was being used. The toxicity was similar to that experienced by humans undergoing the same surgery and needing the same drug. Alley was using a modified dosage, dosing schedule, and infusion rate, which he hoped would dramatically decrease the drug's toxicity. His pilot study suggested that toxicity would be insignificant, but now, with the use of more animals, there was always the potential for a problem.

Alley knew he would have difficulty providing overnight observations of his animals, but he assumed that the IACUC might want to know why this was not occurring, because the first $24 \mathrm{~h}$ after use was the known time frame for the drug's toxicity. However, he provided data from humans and animals that indicated that about $85 \%$ of all toxicity (with the unmodified dosage and schedule) actually occurred within the first $12 \mathrm{~h}$. Based on his pilot study, he didn't think there would be any toxicity at all. Nevertheless, for the first $12 \mathrm{~h}$ he would have somebody observing the animals at least every $30 \mathrm{~min}$. Because recovery from toxicity almost never occurred without significant medical intervention and toxicity treatment was not the objective of the research, he wrote that any animal showing certain defined signs of toxicity would be immediately euthanized.

Also, because the surgery necessitated a thoracotomy, it might seem that someone would have to provide analgesia during the night. To allay that issue, he wrote that the surgery would be performed at about 9 a.m., with the analgesic buprenorphine being administered an hour before the induction of anesthesia. The procedure itself would take only about $15 \mathrm{~min}$, and another analgesic, bupivacaine, would be infiltrated near the surgical incision before closure. The intravenous infusion of the study drug would follow surgery, every $15 \mathrm{~min}$ for the next hour. There would be administered a second dose of buprenorphine in the mid-afternoon (about 3 p.m.), a third dose about 9 p.m., and a fourth dose at about 7 a.m. the following day.

The IACUC debated the need for overnight observations of the rats. One group said that because the study was intended to assess toxicity, there was enough reason to have the animals observed throughout the night and euthanized if necessary. They also felt that there might be a need for an additional dose of buprenorphine during the night. Other members of the IACUC believed that Alley had adequately covered all reasonable contingencies and even had a preliminary study completed to strengthen his position. They said that on the first postoperative day it was not very unusual to have a rat die, whether treated with a potentially toxic drug or not, and if Alley was required to monitor his animals overnight, they might as well do the same for all rodent surgeries.

Did Alley make a sufficiently strong argument, or do you think that there is justification for overnight monitoring for the rats in this study? Are there any other approaches to be considered?

\section{RESPONSE}

\section{Yes (and no)}

\section{Wei-Jeng Chang, PhD}

Should Alley provide overnight monitoring to the rats in his study? The answer is both yes and no.

If the purpose of the experiment is to study the toxic characteristics of the drug and to examine its effects on physiological functions of the rat, then closely monitoring the animals, even at night, is necessary. If the rats required some postoperative medical treatment during the night, such as analgesic administration, then overnight monitoring is necessary.

Otherwise, if the use of the drug is a routine procedure following thoracotomy and the drug causes no toxic effect to the rats, as the literature suggests, then there will be no need for overnight observation of the rats.

In this case, as stated in the first paragraph, Alley has developed a new regimen and requests an animal study to prove his proposal that the drug's toxicity can be dramatically reduced by this regimen. Because no one has ever tested this proposal and it is always possible to discover valuable findings, I would suggest that Alley have someone observe the animals closely and try not to disturb them while entering the animal room especially during the night. To do this he may need some special instrument, such as a videomonitoring system.

Chang is a research fellow at the National Laboratory Animal Center, Taipei, Taiwan.

\section{RESPONSE}

\section{Not in this case}

\section{Rebecca Benz, BA, RLATG \& Matthew L. Dunaif, DVM}

Alley makes a very strong case for foregoing overnight monitoring. He provided data that $85 \%$ of the toxicity occurred within the first $12 \mathrm{~h}$ after treatment, and that was before modifying the dosage and schedule. His pilot study suggested that toxicity would be insignificant were the dosage, dosing schedule, and infusion rate modified. He was observing every $30 \mathrm{~min}$ for the first $12 \mathrm{~h}$ postoperatively and was providing appropriate analgesics.

As members of the veterinary care team, we would like to see some other issues 
addressed before allowing the surgery and drug infusion to occur. First, would it be possible to begin earlier? This would provide more observation time at the end of the day. Second, there is no mention of the route of administration of buprenorphine-intravenous (i.v.), intraperitoneal (i.p.), or subcutaneous (s.c.)? If the administration is i.v., the analgesic effect would occur more quickly and therefore surgery can begin at 8 a.m. instead of 9 a.m. This would also allow for another hour of observation at the end of the day. Third, how many rats undergo the procedure in one day? If it is just one rat, then the time of observation is adequate, but if there are as many as four rats, then the last one is only being observed for $11 \mathrm{~h}$, not 12 .

There is no mention of the route or dosage of the postoperative analgesics. According to Flecknell ${ }^{1}, 0.01-0.05$ $\mathrm{mg} / \mathrm{kg}$ body weight buprenorphine given i.v. or s.c. will last $8-12$ h. The animals are receiving the third dose at 9 p.m. and the fourth at 7 a.m. For a duration of $10 \mathrm{~h}$, either route should be adequate. If the IACUC wanted fur- ther assurance of analgesia, then the rats can receive buprenorphine in the drinking water at $0.006 \mathrm{mg} / \mathrm{ml}$ (ref. 2). The animals should be ambulatory and able to access drinking water before a staff member making observations should leave. A caring animal care staff will not leave an animal in distress at the stroke of 9 p.m. Provisions should be made for the observing staff to be allowed to euthanize an animal that is not ambulatory after 12 $\mathrm{h}$ postoperatively.

With these issues addressed, we believe that Alley should be allowed to conduct his study without overnight observation. In case of any mortality from 9 p.m. to 7 a.m., there should be a necropsy to determine if death was due to drug toxicity or the thoracotomy.

1. Flecknell, P.A. Laboratory Animal Anaesthesia (Academic Press, London, 1996).

2. Deeb, B.J., Eyman, P., Hutton, M.L. \& Abbott, L.C. Efficacy of synthetic opioid analgesics administered in drinking water of rats. $L a b$. Anim. Sci. 39(5), 473 (1989).

Benz is Veterinary Medical Unit Supervisor, and Dunaif is Consulting Veterinary Medical Officer, Medical Research, VA Western New York Healthcare System, Buffalo, NY.

\section{RESPONSE}

\section{Proposal justified}

Kathy Nepote, VMD, MPH

It seems that the researchers at this institution have good training, and it is great that an investigator is anticipating the concerns of the IACUC and taking specific steps to try to alleviate them.

The two primary concerns of the IACUC are postoperative analgesia for the pain associated with the thoracotomy and minimization of animal discomfort from the potential toxic effects of the study drug. Both analgesics, the local (bupivacaine) and the parenteral (buprenorphine), are appropriate to the surgery. The investigator has made a concerted effort to ensure adequate analgesic coverage during the immediate postoperative period. The recommended dosage schedule for buprenorphine is generally every 8-12 h (ref. 1,2), which is consistent with the investigator's proposal. I do not see any reason to ask for an additional injection between 9 p.m. and 7 a.m.

Can there be assurance to the IACUC that the 12-h observation period would 'catch' all rats that would experience toxicity? As with many biological studies, the answer is maybe. Alley's literature citations and his preliminary study appear to justify his plans. What is necessary for the IACUC to be truly comfortable? I would ask for more information about the nature of the toxicity. What clinical signs occur? What is the discomfort that the rats would experience? Would buprenorphine alleviate the pain and discomfort of the toxicity? If so, any rats that experience toxicity beyond the $12 \mathrm{~h}$ would have their pain mitigated. Are there early signs that indicate when a rat might experience toxicity? If so, these signs should be noted in the recorded observations. The IACUC could then mandate that if, after $12 \mathrm{~h}$, any of the rats show these pretoxicity signs, then Alley would have to follow these rats for more than the $12 \mathrm{~h}$. Because rats are more active in the evening (the investigator should make sure the rats have their appropriate 'dark period'), any marked differences in activity should be obvious. This could be one sign that must have monitoring. The investigator's time course for the study with several hours of 'darkness' should facilitate these observations and allow him to observe potential toxicity in the rats.

I believe that Alley has provided sufficient information for the IACUC to accept the 12$\mathrm{h}$ observation period. However, it would be wise to obtain additional information about the toxicity. The IACUC needs to be sure it understands the discomfort the rats might experience. It appears that Alley is taking all reasonable steps to minimize any potential discomfort. However, the IACUC should feel more 'comfortable' with the provision for a longer period of observation should any rat show discomfort or signs of potential toxicity.

1. Swindle, M.M., Vogler, G.A., Fulton, L.K., Marini, R.P. \& Popilskis, S. in Laboratory Animal Medicine 2nd Edn. (eds. Fox, J.G., Anderson, L.C., Loew, F.M. \& Quimby, F.W.) Ch. 22 (Academic Press, New York, 2002).

2. Carpenter, J.W., Mashima, T.Y. \& Rupiper, D.J. (eds.). Exotic Animal Formulary 2nd Edn. (WB Saunders, Philadelphia, 2001).

Nepote is Director, Laboratory Animal Care, University of Maryland, College Park, MD. 